\title{
3D GEO-CLUSTERING FOR WIRELESS SENSOR NETWORK IN SMART CITY
}

\author{
S. Azri ${ }^{1}$, U. Ujang ${ }^{1}$, A. Abdul Rahman ${ }^{1}$ \\ ${ }^{1}$ 3D GIS Research Lab, Faculty of Built Environment and Surveying, 81310 UTM Johor Bahru, Malaysia - (suhaibah, \\ mduznir, alias)@utm.my
}

KEY WORDS: Smart City, Wireless Sensor Network, Spatial Database, Clustering Algorithm, Data Structure

\begin{abstract}
:
Smart city is a connection of physical and social infrastructure together with the information technology to leverage the collective intelligence of the city. Smart cities depend on a great extent on wireless sensor network to manage and maintain their services. Advanced sensor technologies are used to acquire information and help dealing with issues like air pollution, waste management, traffic optimization, and energy efficiency. However, no matter how much smart city may focus on sensor technology, data that are produced from sensors do not organize themselves in a database. Such tasks require a sophisticated database structure to produce informative data output. Besides that, wireless sensor network requires a proper design to improve the energy efficiency. The design will aid to prolong the lifespan of wireless network efficiently. In this study, we proposed a new technique that will be used to organize the information of wireless sensor network in the spatial database. Specific algorithm which is 3D geo-clustering algorithm is used to tackle several issues of location of the sensor in three-dimensional urban area in smart city. The algorithm is designed to minimizing the overlap among group clusters. Overlap plays an important role for energy efficiency. Thus, detection of sensors in two or more group clusters will avoid it from transmitting the same signal to cluster head node. It is prove that this algorithm would only create 5\% to $10 \%$ overlap among group clusters. Several experiments are performed in this study to evaluate the algorithm. Based on the simulation results indicate that this algorithm can balance nodes energy consumption and prolong the network's life span. It also has good stability and extensibility. Several tests are performed to validate the efficiency of the technique to measure the database performance.
\end{abstract}

\section{INTRODUCTION}

Smart city is a term used to define a city that functioning in a intelligent and sustainable way. All the infrastructures and services are integrated and monitored by a smart devices (Giffinger et al., 2007). The increasing number of population has caused the significant impact on resources, climate change and other environmental issues especially in urban areas. According to United Nations (Nations, 2018) By 2050 more than $68 \%$ of world population will live in urban areas. This growth will significantly driving an impact to existing energy resources and climate related changes. Thus, to overcome this issue, smarter infrastructure for greener and energy efficiency in cities is necessary.

According to (P Hancke and Silva, 2012), sensing is the heart of smart infrastructures. It can monitor themselves to monitor public infrastructures in a city such as bridges, roads and buildings. By using real-time monitoring system, it can reduce cost by eliminating the need of regular inspection. Besides that, by measuring energy consumption, it would allow for accurate load forecasting. This is important especially for intelligent system.

Sensor is an object that is able to detect any changes in its surrounding. The change is an input that will be processed and converted into signal. The signal could be transformed into binary (on/off), acceleration or certain value such as temperature, humidity and position. Sensors are operating in a real time. Thus, it could produce large amount of information. The main role of sensor is to acquire information. Sensor is used to help dealing with several issues on safety and security, energy monitoring and control, environmental protection and health (Ho et al., 2005, Patel et al., 2012, Zhang et al., 2017).

A group of spatially dispersed and dedicated sensors is referring as wireless sensor network. In smart city, wireless sensor is the main key role to manage their services. With the help of information and communication technologies, smart city relying a great extent on wireless sensor networks. For example, to aid decision for traffic congestion, information from sensors can be used to identify which lane to close or open to ease congestion. For waste management application, dumpsters can be

\footnotetext{
* Corresponding author
} 
equipped with sensors for route planning and estimated number of staff and trucks needed.

Figure 1 describes the use of sensors in smart city. From the figure we can see that sensors are an integral part for smart city infrastructure. However, no matter how much smart city may focus on sensor technology, data that are produced from sensors do not organize themselves in a database. Such tasks require a sophisticated database structure to produce informative data output. In this paper, new technique that will be used to organize the information of wireless sensor network in the spatial database.

The next section in this paper is discussed problem and motivation regarding smart city and wireless sensor network. In section 3, the concept of the proposed method is explained with its implementation. Section 4 presents the analysis and results of the experiment. Finally, the conclusions are presented in Section 5.

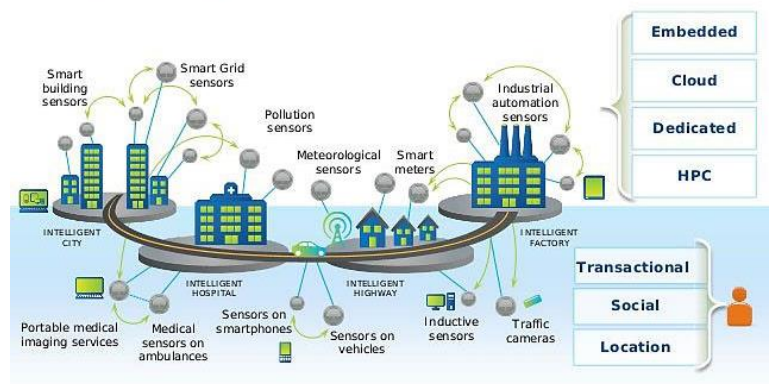

Figure 1. Sensors in smart city.

\section{RESEARCH PROBLEMS AND MOTIVATIONS}

In this section background to the problem on wireless sensor network and clustering techniques are discussed and reviewed. From the problem, proposed technique for $3 \mathrm{D}$ geo-clustering is discuss in the next section.

\subsection{The Architecture of Wireless Sensor Network}

Sensors are one of the significant technologies for smart city. It produces various types of data for different applications. For example, camera sensors return images or even image streams. Smart grid sensors can provide real-time data about grid conditions, detecting outages, faults and load and triggering alarms.

Sensor data is one of the integral components of Internet of Things (IoT). For IoT scenario, each of entity can be outfitted with unique identifier (UID) and capacity to transfer data over a network. Based on (Zaslavsky and Georgakopoulos, 2015) most of the transmitted data are come from sensor. Transmitted data are huge and could produce a lot of information. It is consider as the next challenge for data management.
In a city, sensors are represented as a network that connects to one another. What make them different are wired and wireless networks. In the past few years, networking and operating system communities have giving a lot of attention on wireless sensor network (Baccelli et al., 2013, Dutta and Dunkels, 2012). This is due to easy installation or less hustle especially harsh and hostile environments where wired networks can't be deployed. For example, wireless sensor in a forest can be dropped from the air to avoid going down and setting up wired sensor is not possible.

Most of wireless sensor will operate unattended and unstrapped. The sensor will be battery powered and energy-efficiency will be the main consideration. According to (Pourmirza and Brooke, 2012, Miettinen and Nurminen, 2010) energy cost of communication must be higher than the cost of local computation. This is due to the transportation or transmission of large volumes of data for certain distance that can degrade the network lifetime

In sensor network, node can be defined as a centre that capable to process, gather information from sensor and communicates with other connected nodes in the network. Nodes are supplied with limited battery, limited processor, limited energy, transceiver and small amount of memory. Node could also be homogenous node or heterogeneous node. The position or location of nodes in a network is important. Nodes that close to each other will have more overlap because they share the same environments and tend to receive the same data from sensors. This situation would generate repetitive data entry. Thus it is important to identify nearest nodes for sensor in network so that it will be balance and benefitted for energy cost efficiency.

The characteristic of wireless sensor network described above shows that the structure or the network design is significant. According to (Govindan et al., 2002), sensor networks are best designed as data centric routing systems that use bottom-up mechanism. Meanwhile in database application, data are model as top-down mechanism. However, when the whole network is studied, the possibility to accommodate the design of wireless sensor network in database is straightforward. Figure 2 describes the wireless sensor network with its nodes.

\subsection{Clustering the Wireless Sensor Network}

Data aggregation in wireless sensor network could also help in reducing energy consumption and overhead transmission. Several groups can be used to accommodate sensors and this concept can be applied by using clusters. Network clustering can be defined as the division of nodes in a group cluster. The advantage of clustering to the sensor network is, it can prolong network lifespan 
(Keynes and Punithavathani, 2017, Abbasi and Younis, 2007, Gupta and Pandey, 2016). Besides that, scalability and energy efficiency of the network can be achieved.

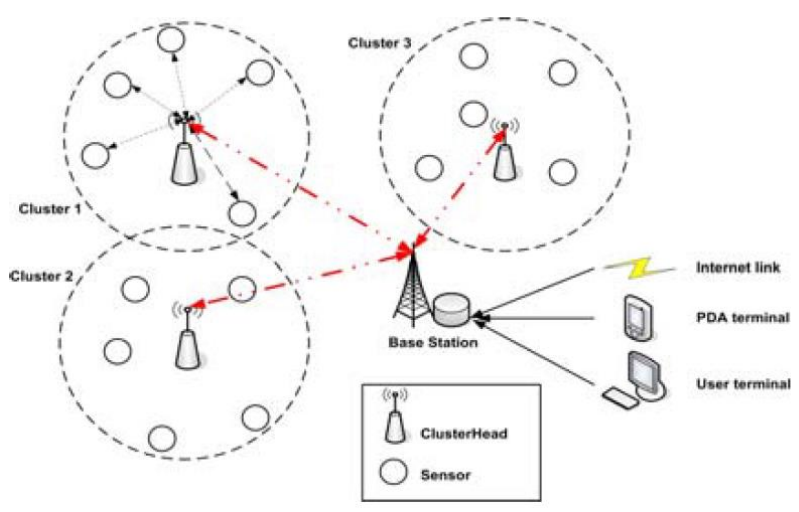

Figure 2. Wireless sensor network and nodes (Dechene et al., 2007).

There are several efforts of using clustering for wireless sensor network. In fact, algorithms are invented for this application, for example, PEGASIS (Power Efficient Gathering in Sensor Information System), LEACH (Low Energy Adaptive Clustering Hierarchy) and LCA (Linked Clustering Algorithm). Details for each algorithm are described as follow:

\subsubsection{PEGASIS}

Power Efficient Gathering in Sensor Information System (PEGASIS) is classified as grid-based topology and sensor are divided into several grids (Lindsey and Raghavendra, 2002). Thus this type of clustering belongs to location-aware routing. A major feature is it can perform without any routing table. PEGASIS implement a near optimal chain-based protocol. It is an improvement over LEACH technique. Each node communicates only with it closest neighbouring node and take turn to transmit signal or information.

\subsubsection{LEACH}

Low-Energy Adaptive Clustering Hierarchy (LEACH) is hierarchical scheme clustering. The main target of this type of clustering is to maintain energy level using multi hop paths (Rhim et al., 2018). By using this scheme, energy used to transmit data can be conserved. LEACH utilizes randomized rotation of cluster-head node to produce evenly load energy in sensor network (Heinzelman et al., 2000). This method use localized coordination for scalability and robustness.

\subsubsection{LCA}

Linked Clustering Algorithm (LCA) is categorized as heuristic algorithm (Singh et al., 2010). The aim of this algorithm is to find reasonable run time and find optimal solution. The search is not depending on particular parameter and such condition may lead to better performance. In LCA, node is identified with an ID number. It has two ways to become cluster head. Node with highest ID number will be chosen as cluster head or if none of the neighbours are cluster head, then it becomes cluster head (Dechene et al., 2007).

However most of the above algorithms are designed for network sensor in 2D. In real world, sensors location is in 3D. It might be placed on a building façade, on top of the tower or on the car roof. Thus, it should be clustered as in 3D. Locating sensors in 2D might cause some error of its real position or might lead to unnecessary decision making. For example, sensors on a building that is represented in 2D might not display all the sensors correctly. Sensors that are overlap with one another will be appeared as one sensor if we represent it in 2D.

Besides that, less focus is given to overlap criteria during the process of group cluster. We believe that this is one of the important steps to avoid repetitive data entry while identifying nearest nodes to communicate. Therefore, in this research we proposed clustering technique in 3D based on location of the sensors. This approach offers minimal overlap among group clusters. Details on the proposed approach are described in the next section.

\section{3D GEO-CLUSTERING}

3D geo-clustering is a technique to cluster sensor data using geographical location in three-dimensional environment. Current approaches for sensor clustering are using 2D clustering technique. The main drawback of this approach is it is not realizing the real location and position of the objects. In real world, sensors are located and positioned in 3D. For example, Closed-Circuit Television (CCTV) also known as video surveillance sensor is located on building façade, sensor on top of telecommunication tower and video image processor on top of vehicle roof. The advantage of using 3D location has been highlighted by (Stoter and Zlatanova, 2003). In their study, 3D gives better understanding to users and decision maker to assist them for decision making. Thus, some information will be loss if its dimension is reduced.

This study proposed 3D geo-clustering to cluster the sensor data. Figure 3 shows the simulation data of several sensors in urban area. Information on sensors and its location are stored in the database. Based on the information from the database, location of sensor data are extracted as a data input for 3D geo-clustering algorithm. Then, all these location are bounded in $3 \mathrm{D}$ parallelepiped 
as a boundary box. To create 3D parallelepiped, minimum and maximum value of $\mathrm{x}, \mathrm{y}$ and $\mathrm{z}$ coordinates are needed.

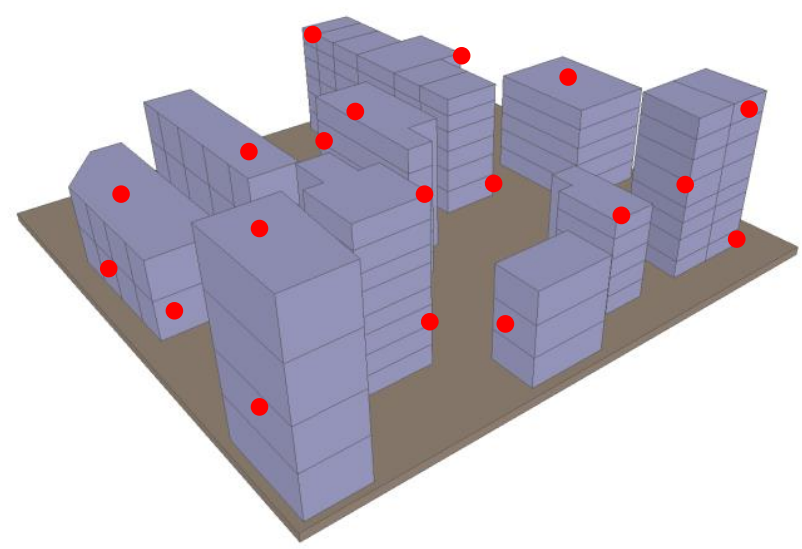

Figure 3. Sensors location in urban area

Then the sensors are classified based on extracted information from database; Less Energy $\left(\mathrm{L}_{\mathrm{E}}\right)$ and Greater Energy $\left(\mathrm{G}_{\mathrm{E}}\right)$. Based on this classification, several sensors with Maximum Greater Energy $\left(\mathrm{G}_{\mathrm{Max}}\right)$ are chosen as Head Node (HeadNode). Then, all sensors are clustered based on its nearest location to HeadNode. If the two or more node with Greater Energy are detected side by side or near to each other in one area, the one with Maximum Greater Energy is chosen as HeadNode and the rest set to sleep mode. At this stage, $k$-means++ algorithm is used to cluster the object. However, cluster centre are set to the sensor with $\mathrm{G}_{\mathrm{Max}}$ energy. Based on previous study by (Suhaibah et al., 2015), $k$-means++ is able to produce minimal overlap among group clusters. The following paragraph explains the details algorithm for 3D geoclustering.

In the Figure 4, 540 sensors are clustered using 3D geoclustering algorithm. Four clusters are generated with four HeadNode (in black color). Analysis on overlapping percentage among clusters shows that only $5 \%$ to $10 \%$ overlap occurs and it is considers minimal overlap.

\begin{tabular}{|c|c|}
\hline $\begin{array}{l}\text { Algorithm } \\
1\end{array}$ & 3D Geo-Clustering for sensors \\
\hline Input $\mathrm{I}$ & $\begin{array}{l}\text { LOCATION OF SENSORS IN 3D, } \\
\text { ENERGY VALUE }\end{array}$ \\
\hline Output & GROUP CLUSTER \\
\hline 1. & $\begin{array}{l}\text { get the min and max point } \\
\text { bounding parallelepiped (in } 3 \mathrm{D}, 3 \\
\text { points are needed to define a } \\
\text { parallelepiped) }\end{array}$ \\
\hline 2. & $\begin{array}{l}\text { find sensor with Less Energy and } \\
\text { Greater Energy }\end{array}$ \\
\hline 3. & classify into group \\
\hline 4. & $\begin{array}{l}\text { choose Max Greater Energy as } \\
\text { Head Node }\end{array}$ \\
\hline
\end{tabular}
5. initialize Head Node as cluster centre $C_{1}, C_{2}, \ldots C_{k} \in \mathbb{R}^{3}$
6. repeat until convergence: \{ for every $i$, set

$$
c^{(i)}:=\arg \min _{j}\left\|x^{(i)}-\mu_{j}\right\|^{2}
$$

for every $j$, set

$$
\mu_{j}:=\frac{\sum_{i=1}^{m} 1\left\{c^{(i)}=j\right\} x^{(i)}}{\sum_{i=1}^{m} 1\left\{c^{(i)}=j\right\}}
$$

7. end if all $C \in C$ completed

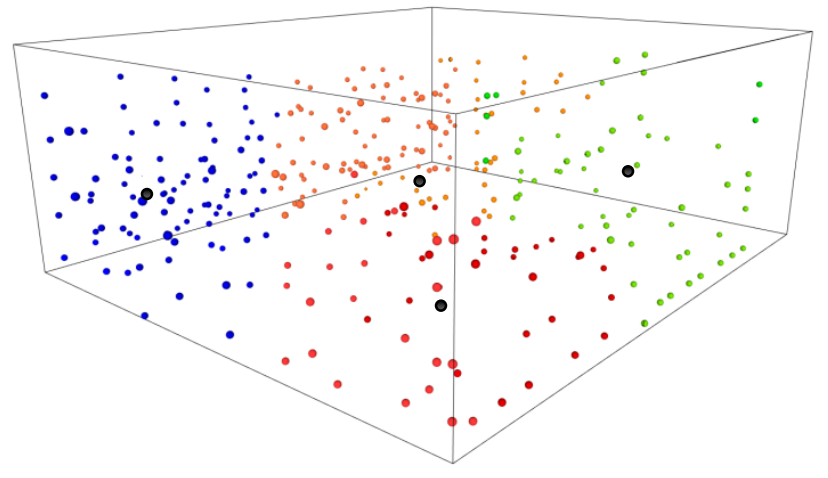

Figure 4. Clustered sensor using 3D geo-clustering.

\section{EXPERIMENT AND ANALYSIS}

Several experiments and analyses are performed in order to verify the proposed $3 \mathrm{D}$ geo-clustering for sensors location.

\subsection{Detection of Overlap}

As mentioned in previous section and paragraphs, overlap plays an important role for energy efficiency. In this experiment, sensor with less energy is detected from group cluster. If this sensor is located in the overlap area of two or more group clusters, it will transfer its information to the nearest HeadNode. Thus, it will save the sensor energy from sending or transmit the information to farthest HeadNode. Based on three groups of sensor location with different numbers, several number of less energy sensors that are located in overlapping cluster area is detected. Figure 5 shows 10 sensor from 200 sensors, 23 sensors from 500 sensors and 48 sensors from 1000 sensors with $\mathrm{L}_{\mathrm{E}}$ are detected in overlap area. This is shows that produced overlap by $3 \mathrm{D}$ geo-clustering is minimal. 


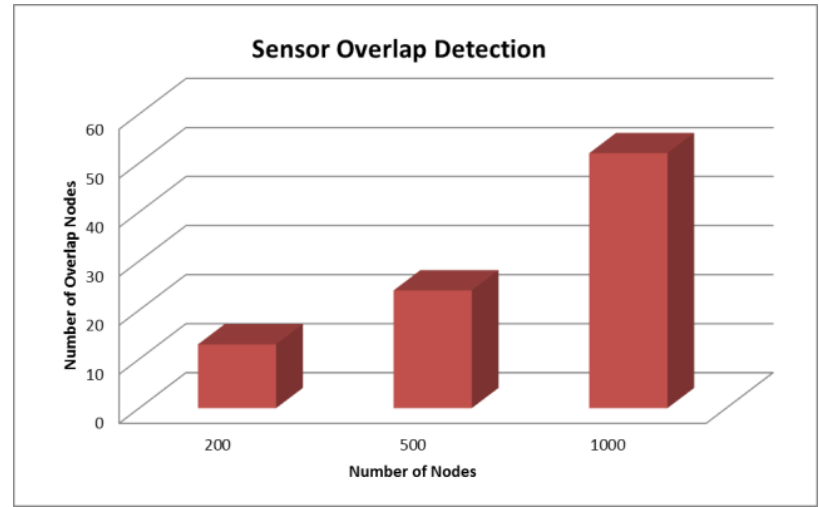

Figure 5. Number of $\mathrm{L}_{\mathrm{E}}$ sensors in overlap area.

\subsection{Detection of Greater Energy Sensor}

Detection of grater energy sensor is important to appoint it as HeadNode. However, during the detection, the sensor location could be located side by side. The possibility of this sensor to be in one cluster is high. Even if the sensors are separated in different group cluster, it will contribute to high cluster overlap. Thus, only one sensor is chosen as HeadNode. The other one is chosen to go to sleep mode for energy saving. Three groups with 200, 500 and 1000 sensors are used in this experiment. The first group detect 35 Greater Energy sensors that are located side by side or closest to each other. Second group detects 48 greater Energy sensors from 500 and the third group detect 78 out of 1000. Figure 6 shows the detection of sensors with greater energy. From the detection, several sensors are set to sleep mode to save the sensors energy. The data for this experiment is tabulated in Table 1. From this experiment, it is shows that more than half number from the detection list are set to sleep.

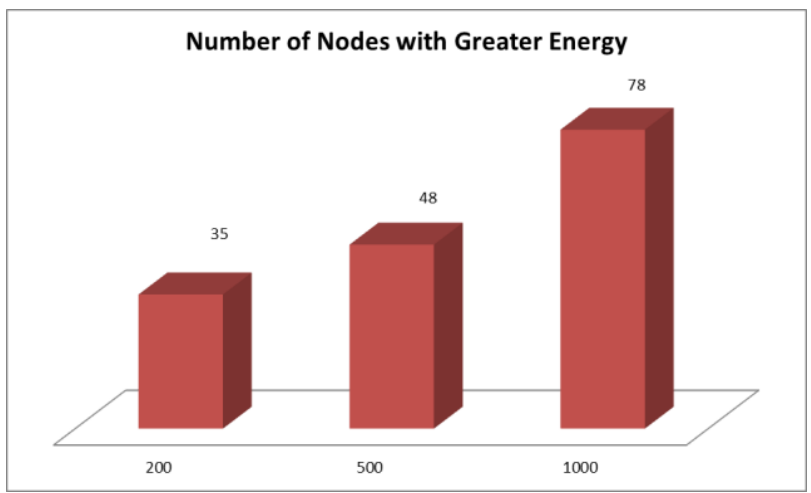

Figure 6. Sensors with greater energy.
Table 1. Number of sensor that are set to sleep mode.

\begin{tabular}{|c|c|c|}
\hline $\begin{array}{c}\text { Number of } \\
\text { Sensors }\end{array}$ & $\begin{array}{c}\text { Greater Energy } \\
\left(\mathrm{G}_{\mathrm{E}}\right) \text { Sensor }\end{array}$ & $\begin{array}{l}\text { Sensor on Sleep } \\
\text { Mode }\end{array}$ \\
\hline 200 & 35 & 25 \\
\hline 500 & 48 & 36 \\
\hline 1000 & 78 & 53 \\
\hline
\end{tabular}

\section{CONCLUSIONS}

This paper proposed 3D geo-clustering algorithm for wireless sensor network (WSN). Using proposed algorithm, sensors are clustered based on its location and clustered based on its energy value. The algorithm is aimed to reduce overlap among clusters to avoid redundant data transmission and identified sensor head node for energy saving.

Based on the test and analysis, 3D geo-clustering algorithm it is shows that detection of overlap cluster is very minimal. Thus only a few sensors are belong to more than one cluster. To save the energy of data transmission, sensors that are belong to more than one clusters are filtered and assigned to one cluster. It will transmit data to one Head Node. Another test shows that, 3D geoclustering can be used to detect greater energy node or sensor. Detection of greater energy sensor is important in the process of choosing Head Node. During the detection, two or more sensors with greater energy that are in the same area or close to each other are filtered. This is to avoid other sensors from transmit data to multiple sensors or to transmit data to farthest Head Node instead of the closest. This procedure could achieve energy efficiency and prolong network life span.

On the basis of these findings we believe that the proposed 3D geo-clustering algorithm is suitable for clustering the wireless sensor network. However, improvement and further testing is suggested to facilitate additional improvements.

\section{REFERENCES}

ABBASI, A. A. \& YOUNIS, M. 2007. A survey on clustering algorithms for wireless sensor networks. Computer communications, 30, 2826-2841.

BACCELLI, E., HAHM, O., GUNES, M., WAHLISCH, M. \& SCHMIDT, T. C. RIOT OS: Towards an OS for the Internet of Things. Computer Communications Workshops (INFOCOM WKSHPS), 2013 IEEE Conference on, 2013. IEEE, 79-80.

DECHENE, D., EL JARDALI, A., LUCCINI, M. \& SAUER, A. 2007. A Survey of Clustering Algorithms for Wireless Sensor Networks.

DUTTA, P. \& DUNKELS, A. 2012. Operating systems and network protocols for wireless sensor networks. Phil. Trans. R. Soc. A, 370, 68-84. 
GIFFINGER, R., FERTNER, C., KRAMAR, H. \& MEIJERS, E. 2007. City-ranking of European medium-sized cities.

GOVINDAN, R., HELLERSTEIN, J., HONG, W., MADDEN, S., FRANKLIN, M. \& SHENKER, S. 2002. The sensor network as a database. Citeseer.

GUPTA, V. \& PANDEY, R. 2016. An improved energy aware distributed unequal clustering protocol for heterogeneous wireless sensor networks. Engineering Science and Technology, an International Journal, 19, 1050-1058.

HEINZELMAN, W. R., CHANDRAKASAN, A. \& BALAKRISHNAN, H. Energy-efficient communication protocol for wireless microsensor networks. Proceedings of the 33rd Annual Hawaii International Conference on System Sciences, 7-7 Jan. 2000 2000. 10 pp. vol.2.

HO, C., ROBINSON, A., MILLER, D. \& DAVIS, M. 2005. Overview of Sensors and Needs for Environmental Monitoring. Sensors, 5, 4.

KEYNES, J. K. D. \& PUNITHAVATHANI, D. S. Clustering methodology to prolong lifetime in wireless sensor networks. 2017 International Conference on Information Communication and Embedded Systems (ICICES), 23-24 Feb. 2017 2017. 1-8.

LINDSEY, S. \& RAGHAVENDRA, C. S. PEGASIS: Powerefficient gathering in sensor information systems. Proceedings, IEEE Aerospace Conference, 9-16 March 2002 2002. 3-3.

MIETTINEN, A. P. \& NURMINEN, J. K. 2010. Energy Efficiency of Mobile Clients in Cloud Computing.

NATIONS, U. 2018. $68 \%$ of the world population projected to live in urban areas by 2050, says UN.

P HANCKE, G. \& SILVA, B. 2012. The Role of Advanced Sensing in Smart Cities.

PATEL, S., PARK, H., BONATO, P., CHAN, L. \& RODGERS, M. 2012. A review of wearable sensors and systems with application in rehabilitation. Journal of NeuroEngineering and Rehabilitation, 9, 21.

POURMIRZA, Z. \& BROOKE, J. 2012. Integration of Wireless Sensor Networks and Local Computational Unit in the Communication Network of the Smart Grid.

RHIM, H., TAMINE, K., ABASSI, R., SAUVERON, D. \& GUEMARA, S. 2018. A multi-hop graph-based approach for an energy-efficient routing protocol in wireless sensor networks. Human-centric Computing and Information Sciences, 8, 30.

SINGH, S. K., SINGH, M. \& SINGH, D. 2010. Energyefficient homogeneous clustering algorithm for wireless sensor network.

STOTER, J. \& ZLATANOVA, S. 3D GIS, where are we standing? ISPRS Joint Workshop on'Spatial, Temporal and multi-dimensional data modelling and analysis', Québec, October, 2003, 2003.

SUHAIBAH, A., FRANÇOIS, A., UZNIR, U., DARKA, M. \& ALIAS, A. R. 2015. Crisp Clustering Algorithm for 3D Geospatial Vector Data Quantization. Springer Verlag.

ZASLAVSKY, A. \& GEORGAKOPOULOS, D. Internet of Things: Challenges and State-of-the-Art Solutions in Internet-Scale Sensor Information Management and Mobile Analytics. 2015 16th IEEE International
Conference on Mobile Data Management, 15-18 June 2015 2015. 3-6.

ZHANG, M., CAO, T. \& ZHAO, X. 2017. Applying SensorBased Technology to Improve Construction Safety Management. Sensors, 17, 1841. 